\title{
Sex-Specific Association of Body Mass Index And Fatty Liver Index With Prevalence of Renal Hyperfiltration: A Cross Sectional Study Using Japanese Health Check-Up Data
}

\section{Atsushi Kitazawa ( $\nabla$ atsukitaaq@gmail.com ) \\ Yoshiharu Fukuda \\ Teikyo University Graduate School of Public Health}

Teikyo University Graduate School of Public Health https://orcid.org/0000-0001-9513-6426

\section{Research article}

Keywords: renal hyperfiltration, BMI, FLI, NAFLD, obesity, BSA, CKD-EPI.

Posted Date: February 8th, 2022

DOI: https://doi.org/10.21203/rs.3.rs-1074104/v1

License: (c) (1) This work is licensed under a Creative Commons Attribution 4.0 International License. Read Full License 


\section{Abstract}

Background: The relationship between obesity and nonalcoholic fatty liver disease and renal hyperfiltration is controversial. This study aimed to assess the correlations of body mass index and fatty liver index, respectively, with renal hyperfiltration in non-diabetic subjects, considering age, sex, and body surface area.

Methods: This cross-sectional study assessed the Japanese health check-up data (FY2018) of 62,379 non-diabetic individuals from a health insurance database. Renal hyperfiltration is the $\geq 95$ th percentile of estimated glomerular filtration rate (derived by Chronic Kidney Disease Epidemiology Collaboration formula) by gender and age in healthy subjects. After adjusting for potential confounders, multiple logistic regression models were applied to evaluate the correlation of renal hyperfiltration with body mass index categories and fatty liver index (10 equal parts).

Results: A negative and positive correlation, respectively, were noted when the body mass index was $<21$ and $\geq 30$ in women; however, a positive correlation was noted for $\mathrm{BMI}<18.5$ and $\geq 30$ in men. Renal hyperfiltration prevalence increased when fatty liver index increased for both sexes; the cutoff value for fatty liver index was 14.7 for women and 30.4 for men.

Conclusions: Body mass index and renal hyperfiltration correlated linearly in women; however, in men, the correlation was U-shaped; therefore, differing by sex. However, fatty liver index correlated linearly with renal hyperfiltration in both sexes. Non-alcoholic fatty liver disease might be associated with renal hyperfiltration; Fatty liver index is a simple marker that can be obtained from health check-ups. Since a high fatty liver index correlated with renal hyperfiltration, it may be beneficial to monitor the renal function in such a population.

\section{Background}

Renal hyperfiltration (RHF) is a well-known phenomenon that occurs early in the development of nephropathy in patients with diabetes [1]. RHF is thought to be followed by the development of proteinuria and progressive decline in renal function [2]. Recently, it has been reported that RHF correlates with prediabetes [3-6], making it an early marker for the onset of diabetes. RHF is associated with renal prognosis and all-cause mortality in patients with diabetes [7].

The following points are important when investigating RHF: 1) the definition of RHF;2) the method of measurement of glomerular filtration rate (GFR); and 3) whether GFR should be adjusted for body surface area (BSA).

1) There is no universal definition of RHF. Some studies have defined RHF as estimated glomerular filtration rate (eGFR) $\geq 120 \mathrm{ml} / \mathrm{min}$ [8], while others have used eGFR to define RHF as the 95th percentile or +2 SD in healthy subjects. According to a systematic review by Cachat et al., $30 \%$ of the studies did not 
justify the choice of the threshold values [9]. From a methodological point of view, they argued that an age-and gender-matched control group should be used to define the RHF threshold.

2) The gold standard for GFR measurement is the inulin clearance test; however, it is not performed in epidemiological studies because it is a complex and time-consuming test. In clinical practice, eGFR, which is estimated from serum creatinine $(\mathrm{Cr})$ values, is used as a measure of GFR. Different formulas are used to determine eGFR across regions and countries; notably, the serum Cr levels vary according to sex, age, race, and other factors. The Modification of Diet in Renal Disease Study (MDRD)-eGFR and Chronic Kidney Disease Epidemiology Collaboration (CKD-EPI)-eGFR formulas are the most commonly used formulas for estimating the GFR. In Japan, the MDRD-eGFR formula has been modified for use in the Japanese population and the modified formula is widely used [10]. For men, eGFR $\left(\mathrm{ml} / \mathrm{min} / 1.73 \mathrm{~m}^{2}\right)$ $=(194 \times \mathrm{Cr}-1.094 \times$ Age-0.287), and for women, it was further multiplied by 0.739. However, the MDRDeGFR equation was developed mainly for chronic kidney disease (CKD) patients; therefore, when it was applied to patients with normal renal function (eGFR $\geq 60$ ), the GFR was estimated to be low in several cases [11]. The CKD-EPI equation was developed to improve on this point by using different equations for estimating the eGFR according to the serum Cr levels ( $\mathrm{Cr} 0.9$ for men and 0.7 for women) [12]. The coefficient in the CKD-EPI formula modified for the Japanese population was 0.813 [13]. The CKD-EPI equation has been noted to be a superior surrogate marker of GFR in patients with hyperfiltration [14]; additionally, the majority of studies on RHF based on eGFR used the CKD-EPI formula. However, studies on Japanese subjects are limited [4-6], and all of the studies used the MDRD-eGFR formula.

3) There is a clinicopathological syndrome associated with obesity called obesity-related glomerulopathy (ORG). The histological feature is glomerulomegaly, which may be due to increased metabolic demand, and functionally, there is an increase in the total glomerular filtration rate [15]. ORG has also been postulated to be a kidney lesion caused by metabolic syndrome. However, studies evaluating the relationship between obesity and RHF are controversial because the results vary depending on whether the GFR is indexed with BSA. Most previous RHF studies that evaluated GFR have found a positive relationship between the BMI and RHF that disappears upon adjustment of GFR to BSA [16-18]. The indexed GFR with BSA in obese individuals may underestimate the GFR. There are few large cohort studies of RHF using estimated GFR that have evaluated its correlation with BMI.

In addition to RHF, non-alcoholic fatty liver disease (NAFLD) is an independent risk factor for cardiovascular diseases. Recently, apart from the general cardiorenal risk factors, such as obesity, hypertension, diabetes, and hyperlipidemia, a strong association between the presence and severity of NAFLD and the prevalence and incidence of CKD has been clarified [19]. It has been suggested that insulin resistance may be a common pathogenic mechanism in NAFLD and CKD [20]. However, only one study has indicated an association between NAFLD (diagnosed by ultrasound or MRI) and RHF [21]. In that study, eGFR was converted to absolute value $(\mathrm{mL} / \mathrm{min})$ using the following equation: (eGFR $\mathrm{mL} / \mathrm{min} / 1.73 \mathrm{~m}^{2}$ * BSA) $/ 1.73 \mathrm{~m}^{2}$. BSA was calculated using the DuBois and DuBois formula (BSA = $0.007184 \times$ Weight $^{0.425} \times$ Height $^{0.725}$ ) [22]. Patients with NAFLD presented higher levels of eGFR and a 
significantly increased prevalence of hyperfiltration (73.2\%) compared to the patients without NAFLD. Moreover, NAFLD and increased weight were associated with an increased probability of hyperfiltration.

The diagnosis of NAFLD is usually made by ultrasonography; however, as a simpler marker, the fatty liver index (FLI), which can be calculated from the BMI, waist circumference (WC), triglyceride (TG), and gamma-glutamyl transferase (GGT) was reported by Bedgni et al. [23], and validation studies have been carried out in each region since it was first reported. In addition, there have been several studies showing that FLI is not only a marker for NAFLD, but also a predictive marker for diabetes and CKD. However, the relationship between FLI and RHF has so far been reported in only one small cohort study in Finnish men [24]. In that study, no correlation was noted between the FLI and RHF; both were independently associated with all-cause and cardiovascular mortality.

The aim of the present study was to assess the correlations of BMI and FLI with RHF in non-diabetic subjects, taking into account the age, sex, and BSA. For the purpose of this study, RHF was defined as the 95th percentile or higher of CKD-EPI eGFR by sex and age in healthy subjects at health check-ups. In addition, the analysis was also adjusted for BSA.

\section{Methods}

\section{Study Design and Data Source}

The present study was a cross-sectional study performed using the Japanese health check-up data pertaining to FY2018. The data were obtained from a health insurance association and comprised annual health check-up data collected from all prefectures in Japan other than Tokyo.

\section{Study Subjects}

The subjects were those aged 40-59 years who underwent a specific health check-up between April 2018 and March 2019. The eligible subjects for this study were those who (1) had all relevant data related to $\mathrm{Cr}$, $\mathrm{HbA1c}$ (based on National Glycohemoglobin Standardization Program units), fasting plasma glucose (FPG), high-density lipoprotein cholesterol (HDL-c), low-density lipoprotein cholesterol (LDL-c), TG, GGT, systolic blood pressure (SBP), diastolic blood pressure (DBP), weight, height, and WC; (2) had no cardiovascular disease, chronic kidney disease, or stroke according to the questionnaire of the health check-up at FY2018; (3) did not have diabetes (HbA1c $\geq 6.5 \%$ or FPG $\geq 126 \mathrm{mg} / \mathrm{dL}$ or use of antidiabetics) in FY2018; and (4) had no outlier data for Cr, HbA1c, FPG, LDL, HDL, TG, GGT, weight, height, WC, SBP, or DBP at FY2018. The subjects who met all eligibility criteria are shown in Figure 1.

\section{Definitions of renal hyperfiltration and hypofiltration}

The serum $\mathrm{Cr}$ levels were measured using enzymatic methods. The GFR was estimated from serum $\mathrm{Cr}$ values using the CKD-EPI formula [12] and adjusted using the Japanese coefficient, 0.813 [13]. The value of eGFR derived using this formula was indexed by BSA using the DuBois and DuBois formula [22]. To 
define hyperfiltration and hypofiltration, we focused on "healthy subjects" who met the following criteria: (1) No medication for hypertension, hyperlipidemia, or diabetes mellitus; (2) FPG < $100 \mathrm{mg} / \mathrm{dL}, \mathrm{HbA} 1 \mathrm{c}<$ $5.7 \%, \mathrm{SBP}<120 \mathrm{mmHg}, \mathrm{DBP}<80 \mathrm{mmHg}, \mathrm{LDL}<140 \mathrm{mg} / \mathrm{dl}, \mathrm{HDL} \geq 40 \mathrm{mg} / \mathrm{dl}$, and TG $<150 \mathrm{mg} / \mathrm{dl}$; and (3) a negative urine protein test. The "healthy subjects" were stratified into 8 groups according to sex and age (40-44, 45-49, 50-54, and 55-59 years), and hypofiltration and hyperfiltration were defined as values below the 5th percentile and above the 95th percentile of eGFR in each group, respectively. In addition, using the reference values, all subjects were divided into hypofiltration, normal filtration, and hyperfiltration groups based on their individual eGFR values. Subsequently, the subjects of the RHF were then compared with the subjects of normal filtration. A graph of the reference values for renal hyperfiltration and hypofiltration in women and men is shown in Figure 2.

\section{Variables}

For background variables, age, BMI, FLI, FPG, SBP, DBP, HDL-c, LDL-C, TG, and self-administered questionnaire (antihypertensive medication use, lipid-lowering medication use, current smoking, daily drinking, exercising for 30 minutes or more per day, skipping breakfast, midnight eating, weight gain of 10 $\mathrm{kg}$ or more since 20 years of age, and adequate sleeping) at FY2018 were extracted from the database. The BMI was calculated as the weight divided by height in square meters, and the FLI score was

calculated as follows: $F L I=\left\{\frac{e^{0.953 \times \log (T G)+0.139 \times B M I+0.718 \times \log (\gamma-G T P)+0.053 \times W C-15.745}}{1+\left(e^{0.953 \times \log (T G)+0.139 \times B M I+0.718 \times \log (\nu-G T P)+0.053 \times W C-15.745}\right)}\right\} \times 100$

\section{Statistical Analysis}

For descriptive statistics of the baseline characteristics, the median (IQR) was calculated for continuous variables, and frequency and percentage were calculated for categorical variables for the normal and RHF groups.

Univariate and multivariate analyses were performed by sex to assess the correlation between background and RHF. Multivariate adjusted logistic regression models were subsequently applied to evaluate the correlation of RHF with BMI categories ( $<18.5,18.5 \geq$ to $<20,20 \geq$ to $<21,21 \geq$ to $<23,23$ $\geq$ to $<25,25 \geq$ to $<30$, and $\geq 30$ ) and FLI (10 equal parts), respectively. Multivariate analyses with three models were performed to calculate the odds ratios (ORs) and $95 \%$ confidence intervals (Cls). The models were adjusted for age (categorised into four age groups: 40-44, 45-49, 50-54 and 55-59 years) for Model-1; Model-1 plus FPG level (normal; FPG $<100 \mathrm{mg} / \mathrm{dl}$, prediabetes 1; FPG 100-109 mg/dl, prediabetes 2; FPG 110-125 mg/dl), blood pressure level (Normal; SBP $<120 \mathrm{mmHg}$ and DBP $<80$ $\mathrm{mmHg}$, prehypertension; SBP 120-139 mmHg or DBP $80-89 \mathrm{mmHg}$, hypertension; SBP $\geq 140 \mathrm{mmHg}$ or $\mathrm{DBP} \geq 90 \mathrm{mmHg}$ ), HDL, antihypertensive medication use, lipid-lowering medication use, current smoking, and daily drinking for Model-2; Model-2 plus, exercising for 30 minutes or more per day, skipping breakfast, midnight eating, weight gain of $10 \mathrm{~kg}$ or more since 20 years of age, and adequate sleeping for Model-3. To evaluate the fitness of the model, we performed a lack-of-fit test [25]. 
All the tests were two-tailed, and the significance level was set to 0.05 . For statistical analysis, JMP® version 15.0 (SAS Institute Inc., Cary, NC, USA) was used. The results are reported in accordance with the recommendations of the Strengthening the Reporting of Observational Studies in Epidemiology (STROBE) checklist [26].

\section{Results}

\section{Study population}

Of the 215,063 beneficiaries, data of 62,379 eligible subjects were extracted from the database.

\section{Baseline characteristics}

Descriptive analyses for the baseline characteristics of the eligible RHF and normal filtration subjects by BMI class (lower 18.5, 18.5- $<21,21-<23,23-<25,25-<30$, and 30 or higher) are shown in Tables 1a and $1 \mathrm{~b}$ for women and men. Every variable differed significantly among the groups.

Descriptive analyses for the baseline characteristics of the eligible RHF and normal filtration subjects by 10 equal parts of $\mathrm{FLI}$ are shown in Tables $2 \mathrm{a}$ and $2 \mathrm{~b}$ for women and men, respectively.

\section{Association of RHF and BMI or FLI level}

The results of the multivariate adjusted logistic regression analysis for the three models are presented in Table 3. In women, negative correlation was noted for $\mathrm{BMI}<21$, and positive correlation was noted for $\mathrm{BMI} \geq 30$ for RHF in all the three models.

On the other hand, in men, $\mathrm{BMI}<18.5$ and $\mathrm{BMI} \geq 30$ were positively correlated with $\mathrm{RHF}$ in all three models.

In all the three models, there was a positive correlation of RHF with FLI values above 14.1 in women and above 35.9 in men.

The results of the multivariate adjusted logistic regression analysis for BMI or FLI in Model $3 \S$ are shown in Figure 3.

\section{Cut-off values of FLI for RHF by sex}

The cut-off values for FLI in RHF were 14.7 and 30.4 for women and men, respectively.

\section{Discussion}

In this study, we examined the correlation between BMI or FLI with RHF, respectively. For BMI, there was a positive correlation with RHF in women and a U-shaped correlation in men. On the other hand, there was a linear positive correlation with RHF in both men and women. 
The association between $\mathrm{BMI}$ and RHF differed according to sex. In this analysis, $\mathrm{BMI} \geq 30$ was correlated with RHF in both sexes, which supports previous reports that obesity is a risk factor for RHF [27]. One result not seen in previous reports was that among men, the lean body mass index $(\mathrm{BMI})<18.5$ was also associated with RHF. Although the relationship between low body mass and RHF has not been fully explained, it is widely known that the relationship between BMI and all-cause mortality is U-shaped, with the lowest rates between 22.5 and $25 \mathrm{~kg} / \mathrm{m}^{2}$ [28]. Thus, low or high values of BMI are associated with an increased risk of mortality. Furthermore, several previous studies have argued that RHF is associated with mortality risk $[7,24]$. Therefore, the relationship between low body mass and RHF may reflect a completely different pathology than the relationship between obesity and RHF.

It has been found that even in the absence of diabetes, high insulin resistance is likely to increase the renal intraglomerular hydrostatic pressure [29]. It is known that Asians, particularly East Asians, have a lower capacity for fat storage in subcutaneous adipose tissue, compared with other ethnic groups [30]. Therefore, lipid spillover, in which free fatty acid (FFA) overflow from adipose tissue, is thought to be more likely. Lipid spillover may result in the accumulation of ectopic fat, such as fatty liver, which may lead to insulin resistance. Kadowaki et al. evaluated the fat distribution, adipose tissue insulin resistance, and skeletal muscle insulin resistance in non-obese Japanese men [31]. Even among non-obese individuals, visceral and hepatic fat accumulations were observed in some individuals, with various accumulation patterns. Even in the absence of visceral fat accumulation, muscle insulin resistance (metabolic disturbance) was observed in the presence of fatty liver, whereas no insulin resistance was observed in the absence of fatty liver, even in the presence of visceral fat accumulation. It is notable that extremely thin people have lower muscle mass and are more likely to develop insulin resistance, which may lead to the development of RHF.

In terms of the relationship between FLI and RHF, it was linearly significant from Q8 for women (FLI > 14.1) and Q7 for men (FLI > 31.4). In addition, the cut-off values for FLI in RHF were 14.7 for women and 30.4 for men. The cut-off value of FLI for NAFLD in Asians is about 30; specifically, it is 35 for men and 20 for women [32, 33], which is generally consistent with the present results. It can be mentioned that FLI correlates well with RHF, and NAFLD and RHF might be associated. The FLI might be more useful than BMI in screening for RHF. However, cross-sectional epidemiological studies are not suitable for estimating the pathophysiology or causality. Therefore, further longitudinal studies and interventional trials are needed to further investigate the U-shaped association between RHF and BMI.

This study had several limitations. Because this study was cross-sectional, it was not possible to assume a causal relationship with RHF. There might have been some bias towards the participants who were particularly motivated to undergo a health check-up. Since most of the health check-up recipients are workers, a sampling bias due to the healthy worker effect is possible.

\section{Conclusions}


The BMI and RHF correlated linearly in women, but the correlation was U-shaped in men. On the other hand, FLI and RHF correlated linearly in both sexes. NAFLD may be associated with RHF. FLI is a simple marker that can be obtained from health check-ups. Since a high FLI correlated with RHF, it may be beneficial to monitor the renal function in such a population.

\section{List Of Abbreviation}

RHF; renal hyperfiltration

$\mathrm{BMl}$; body mass index

FLI; fatty liver index

NAFLD; non-alcoholic fatty liver disease

BSA; body surface area

GFR; glomerular filtration rate

CKD; chronic kidney disease

MDRD; Modification of Diet in Renal Disease Study

CKD-EPI; Chronic Kidney Disease Epidemiology Collaboration

SBP; systolic blood pressure

DBP; diastolic blood pressure

HDL-c; high-density lipoprotein cholesterol

LDL-c; low-density lipoprotein cholesterol

TG; triglyceride

GGT; gamma-glutamyl transferase

WC; waist circumference

FPG; fasting plasma glucose

Cr; creatinine

\section{Declarations}


The Teikyo University Ethical Review Board for Medical and Health Research Involving Human Subjects approved this study after due ethical consideration (approval No.:18-200-3).

\section{Consent for publication}

In this study, only anonymised data were used, and we had no access to personal information.

\section{Availability of data and materials}

Data cannot be shared publicly because of agreement between data holder. Data are available from The Mutal Aid Association of Prefectural Govermment Personnel (contact via https://www.chikyosai.or.jp/) for researchers who meet the criteria for access to confidential data.

\section{Competing interests}

The authors declare that they have no competing interests.

\section{Funding}

Funding Information is not available.

\section{Authors' contributions}

AK designed and analyzed the study and interpreted the results. YF edited the study design and interpretation of the results and was a major contributor in writing the manuscript. All authors read and approved the final manuscript.

\section{Acknowledgements}

We thank Honyaku Center Inc. (https://www.honyakucenter.jp/) for editorial support.

\section{References}

1. Helal, I., Fick-Brosnahan, G. M., Reed-Gitomer, B et al. Glomerular hyperfiltration: definitions, mechanisms and clinical implications. Nature reviews. Nephrology 8, 293-300, 2012.

2. Ruggenenti P, Porrini L E, Gaspari $F$ et al. Glomerular hyperfiltration and renal disease progression in type 2 diabetes. Diabetes Care. 2012 Oct;35(10):2061-8.

3. Antonio Rodriguez-Poncelas, Gabriel Coll-de-Tuero, Jordi Blanch et al. Prediabetes is associated with glomerular hyperfiltration in a European Mediterranean cohort study. J Nephrol. 2018 Oct;31(5): 743-749.

4. Okada R, Wakai $\mathrm{K}$, Naito $\mathrm{M}$ et al. Renal hyperfiltration in prediabetes confirmed by fasting plasma glucose and hemoglobin A1c. Ren Fail. 2012;34(9):1084-90. 
5. Okada R, Yasuda Y, Tsushita K et al. Glomerular hyperfiltration in prediabetes and prehypertension. Nephrol Dial Transplant. 2012 May;27(5):1821-5.

6. Kawata I, Koshi T, Hirabayashi K et al. Prediabetes defined by the International Expert Committee as a risk for development of glomerular hyperfiltration. Acta Diabetol. 2019 May;56 (5): 525-529.

7. Giuseppe Penno, Emanuela Orsi, Anna Solini et al. Renal hyperfiltration is independently associated with increased all-cause mortality in individuals with type 2 diabetes: a prospective cohort study BMJ Open Diabetes Res Care. 2020 Jul;8(1): e001481.

8. Sunder-Plassmann, G.; H rl, W.H.; Levey, A.S.; Coresh, J. A Critical Appraisal for Definition of Hyperfiltration (Multiple Letters). Am. J. Kidney Dis. 2004, 396-397.

9. Francois Cachat, Christophe Combescure, Michel Cauderay et al. A Systematic Review of Glomerular Hyperfiltration Assessment and Definition in the Medical Literature. Clin J Am Soc Nephrol. 2015 Mar 6;10(3):382-9.

10. Revised equations for estimated GFR from serum creatinine in Japan. Am J Kidney Dis. 2009 Jun;53(6):982-92.

11. Fontsere' N, Salinas I, Bonal J et al. Are prediction equations for glomerular filtration rate useful for the long-term monitoring of type 2 diabetic patients? Nephrol Dial Transplant 21: 2152-2158, 2006

12. Levey AS, Stevens LA, Schmid CH, et al; CKD-EPI (Chronic Kidney Disease Epidemiology Collaboration). A new equation to estimate glomerular filtration rate. Ann Intern Med. 2009;150(9):604-612.

13. Masaru Horio, Enyu Imai, Yoshinari Yasuda et al. Modification of the CKD epidemiology collaboration (CKD-EPI) equation for Japanese: accuracy and use for population estimates. Am J Kidney Dis. 2010 Jul;56(1):32-8.

14. Stevens LA, Schmid CH, Greene T et al. Comparative performance of the CKD Epidemiology Collaboration (CKD-EPI) and the Modification of Diet in Renal Disease (MDRD) Study equations for estimating GFR levels above $60 \mathrm{~mL} / \mathrm{min} / 1.73$ m2. Am J Kidney Dis 56: 486-495, 2010

15. Denic A, Glassock RJ. Obesity-Related Glomerulopathy and Single-Nephron GFR. Kidney Int Rep. 2020 Jun 2;5(8):1126-1128.

16. Wuerzner G, Pruijm M, Maillard M et al. Marked association between obesity and glomerular hyperfiltration: a crosssectional study in an African population. Am J Kidney Dis. 2010;56(2):30312.

17. Kwakernaak AJ, Toering TJ, Navis G. Body mass index and body fatdistribution as renal risk factors: a focus on the role of renal haemodynamics. Nephrol Dial Transplant. 2013;28 Suppl 4: iv42-49.

18. Kwakernaak AJ, Zelle DM, Bakker SJ et al. Central body fat distribution associates with unfavorable renal hemodynamics independent of body mass index. J Am Soc Nephrol. 2013;24(6):987-94.

19. Christopher D. Byrne, Giovanni Targher. NAFLD as a driver of chronic kidney disease. J Hepatol. 2020 Apr;72(4):785-801. 
20. Giovanni Targher, Michel B Chonchol, Christopher D Byrne. CKD and nonalcoholic fatty liver disease. Am J Kidney Dis. 2014 Oct;64(4):638-52.

21. Manuela Abbate, Non-Alcoholic Fatty Liver Disease Is Associated with Kidney Glomerular Hyperfiltration in Adults with Metabolic Syndrome.

22. D Du Bois, E F Du Bois. A formula to estimate the approximate surface area if height and weight be known. 1916. Nutrition. Sep-Oct 1989;5(5):303-11; discussion 312-3.

23. Bedogni G, Bellentani S, Miglioli L et al. The Fatty Liver Index: a simple and accurate predictor of hepatic steatosis in the general population. BMC Gastroenterol. 2006 Nov 2; 6: 33.

24. Mounir Ould Setti, Ari Voutilainen, Tomi-Pekka Tuomainen et al. Epidemiol Health. 2021; 43 : e2021001. Renal hyperfiltration, fatty liver index, and the hazards of all-cause and cardiovascular mortality in Finnish men.

25. Agresti, A. Categorical Data Analysis. 3rd ed. Hoboken, NJ: John Wiley \& Sons, 2013.

26. von Elm E, Altman DG, Egger $M$ et al. STROBE Initiative. The Strengthening the Reporting of Observational Studies in Epidemiology (STROBE) statement: guidelines for reporting observational studies. J Clin Epidemiol. 2008;61:344-9.

27. Vidar Tor Nyborg Stefansson, Jørgen Schei, Trond Geir Jenssen et al. Central obesity associates with renal hyperfiltration in the non-diabetic general population: a cross-sectional study. BMC Nephrol. 2016 Nov 10;17(1):172.

28. Whitlock G, Lewington S, Sherliker P et al. Body-mass index and cause-specific mortality in 900000 adults: collaborative analyses of 57 prospective studies. Lancet 2009,373:1083-1096.

29. Akihiro Tsuda, Eiji Ishimura, Hideki Uedono et al. Association of Albuminuria With Intraglomerular Hydrostatic Pressure and Insulin Resistance in Subjects With Impaired Fasting Glucose and/or Impaired Glucose Tolerance. Diabetes Care 2018 Nov; 41(11): 2414-2420

30. Nazare JA, Smith JD, Borel AL, Haffner SM et al. Ethnic influences on the relations between abdominal subcutaneous and visceral adiposity, liver fat, and cardiometabolic risk profile: the International Study of Prediction of Intra-Abdominal Adiposity and Its Relationship With Cardiometabolic Risk/Intra-Abdominal Adiposity. Am J Clin Nutr. 2012; 96 (4): 714-726

31. Kadowaki S, Tamura Y, Someya Y et al. Fatty Liver Has Stronger Association With Insulin Resistance Than Visceral Fat Accumulation in Nonobese Japanese Men. J Endocr Soc. 2019 May 20; 3 (7): $1409-1416$

32. Huang $X, X u M$, Chen $Y$ et al. Validation of the Fatty Liver Index for Nonalcoholic Fatty Liver Disease in Middle-Aged and Elderly Chinese. Medicine (Baltimore). 2015 Oct; 94 (40): e1682.

33. Yang BL, Wu WC, Fang KC et al. External validation of fatty liver index for identifying ultrasonographic fatty liver in a large-scale cross-sectional study in Taiwan. PLoS One. 2015 Mar 17; 10 (3): e0120443.

\section{Tables}


Tables 1-3 are available in the Supplemental Files section.

\section{Figures}

\section{Figure 1}

Flow of eligible subjects.

\section{Figure 2}

Distribution of eGFR (CKD-EPI) in "healthy subjects". The 95th and 5th percentiles are shown in 5-year age groups. Hyperfiltration was defined as an eGFR over the age-and sex-specific 95th percentile and hypofiltration was defined as an eGFR below the 5th percentile.

\section{Figure 3}

Graph of correlation between BMI and FLI for RHF. The dots represent each odds ratio for the categories classified by BMI values; additionally, the error bars represent $95 \% \mathrm{Cl}$ of the odds ratio.

\section{Supplementary Files}

This is a list of supplementary files associated with this preprint. Click to download.

- Table1aandb.pdf

- Table2aandb.pdf

- Table3.pdf 